\title{
Consideraciones acerca de la implementación de las acciones sobre el cambio climático y los acuerdos internacionales para la cooperación bajo la perspectiva del Informe Stern
}

\section{Considerations on the Implementation of the Actions on Climate Change and the International Agreements for Cooperation Under the Perspective of the Stern Review}

Jessica Karina Obeso Cuadra, ${ }^{*}$ María Isabel Medrano Sánchez, ${ }^{* *}$ Geraldo Morón Paredes, ${ }^{* * *}$ José Luis Masías Vidal, ${ }^{* * * *}$ Wendy Lidia Moreano Márquez, ${ }^{* * * * *}$ Eugenia García Segovia, ${ }^{* * * * * *}$ Raúl Rosadio Bernal ${ }^{* * * * * *}$

DOI: http://dx.doi.org/10.21503/lex.v16i21.1552

* Abogada. Magíster en Derecho de la Minería, especialización en Derecho Ambiental y Derecho Minero e Hidrocarburos, docente de la Facultad de Derecho y Ciencia Política de la Universidad Alas Peruanas de los cursos Derecho Minero y Derecho del Medio Ambiente.

Correo electrónico: jessicaobesoc@gmail.com

** Abogada. Magíster en Derecho de la Minería. Extensión universitaria internacional en Harvard University. Docente de la Facultad de Derecho y Ciencia Política de la Universidad Alas Peruanas del curso Derecho Minero e Hidrocarburos y coordinadora de la Mención de Minería y Medio Ambiente.

Correo electrónico: isabel@medrano.pe ; m_medrano@doc.uap.edu.pe

*** Ingeniero metalúrgico. Segunda especialización en Medio Ambiente, Desarrollo Sostenible y Recursos Naturales. Especialización en Alta Gerencia. MBA. Expositor de diplomados y cursos de especialización en Medio Ambiente, Desarrollo Sostenible, Seguridad y Sistemas Integrados de Gestión a nivel de postgrado.

Correo electrónico: gmph26@hotmail.com

**** Ingeniero ambiental. Maestro en Docencia Universitaria y Gestión Educativa. Especialista en Monitoreo y Evaluación de la Calidad Ambiental y en Seguridad y Salud en el Trabajo. Coordinador académico y docente de la Escuela de Ingeniería Ambiental de la Universidad Alas Peruanas. Correo electrónico: j_masias@uap.edu.pe

***** Ingeniera civil con maestría en Ingeniería Civil con mención en Gestión y Gerencia en la Construcción. Egresada de la maestría en Ingeniería Vial y doctoranda en Medio Ambiente y Desarrollo Sostenible. Experiencia en el sector público en temas de transportes y gestión vial en vías nacionales.

Correo electrónico: wendymoreanom@hotmail.com

****** Arquitecta con estudios de maestría en Gestión Pública, delegada titular por parte del Colegio de Arquitectos del Perú en la Comisión Técnica de Edificaciones del Centro Histórico de Lima, docente de la Facultad de Arquitectura y Urbanismo de la Universidad Privada del Norte de los cursos de Urbanismo Sostenible II y III

Correo electrónico: mariaeugenialourdes@yahoo.com

***** Demógrafo, magíster en Ecología General y doctor en Medio Ambiente y Desarrollo Sostenible. Profesor principal de la Facultad de Ciencias Naturales y Matemáticas y de Postgrado en la UNFV. Correo electrónico: rosbern7@hotmail.com

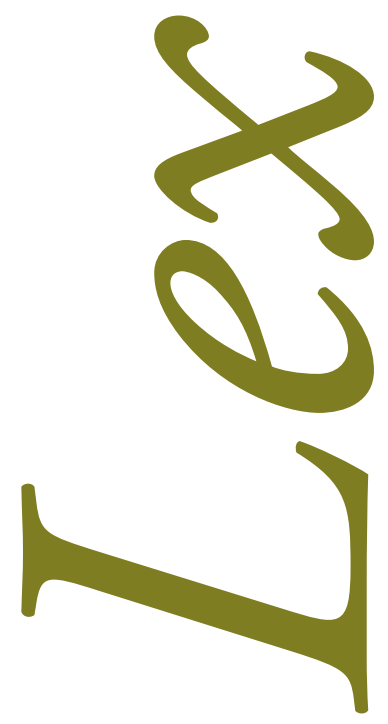




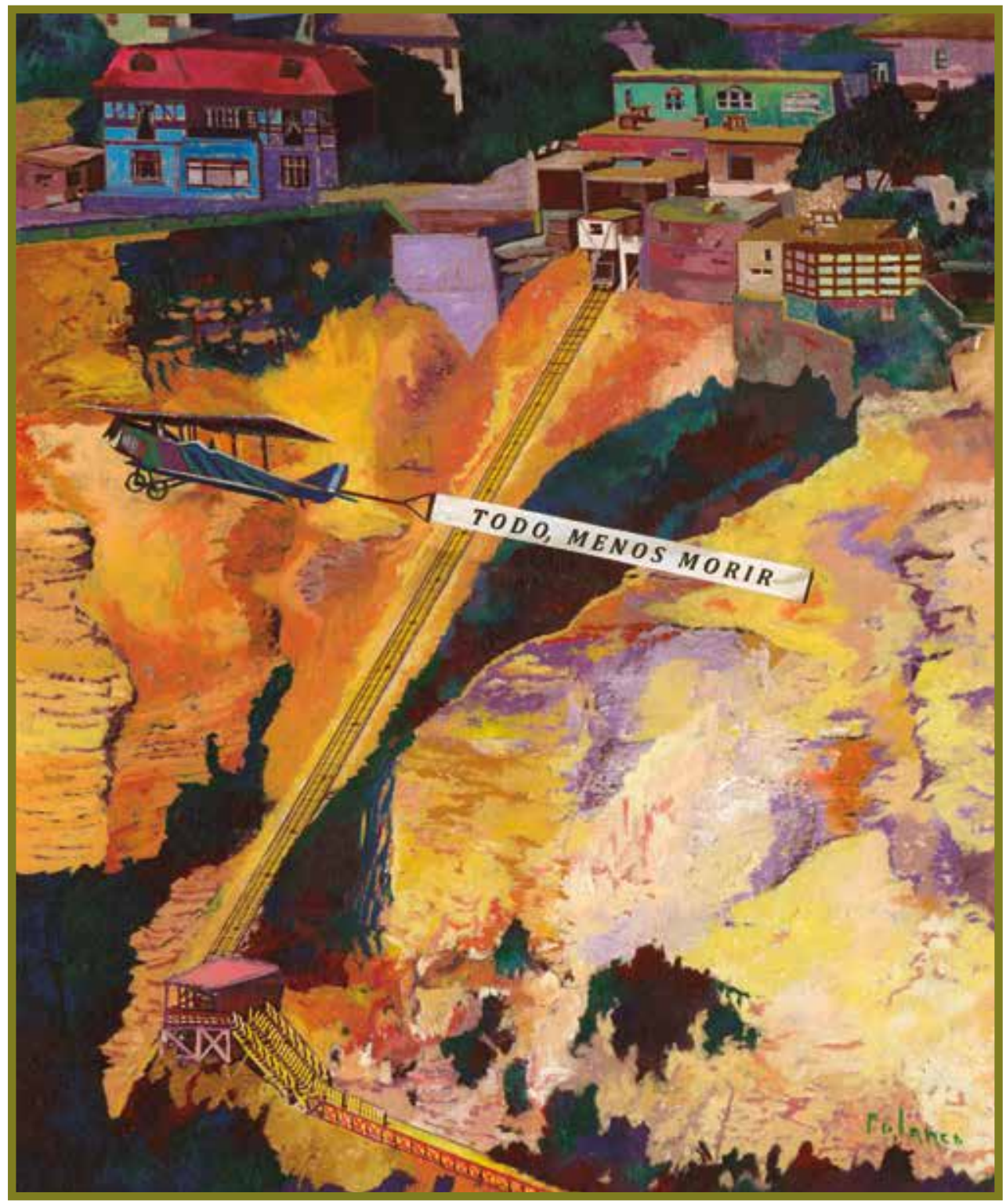

Todo menos morir. Enrique Polanco.

https://www.facebook.com/enriquepolancopintor/ 


\section{RESUMEN}

El Informe Stern sobre la Economía del Cambio Climático (Stern Review on the Economics of Climate Change) es un documento redactado por el economista Sir Nicholas Stern, antiguo miembro del Banco Mundial por encargo del Tesoro Británico, sobre el impacto del cambio climático y el calentamiento global sobre la economía mundial al afirmar que se necesita de una inversión equivalente al $1 \%$ del PIB mundial para mitigar los efectos del cambio climático; caso contrario, el universo estaría expuesto a una recesión que podría alcanzar al $20 \%$ del PIB. Se trata de un informe político riguroso que logró conseguir la atención de la opinión pública sobre las implicancias y consecuencias del cambio climático, dejándonos conclusiones claras y contundentes sobre una pronta actuación. Su discusión trata sobre cómo mitigar el cambio climático y si estamos preparados para este proceso desde el enfoque económico. El informe presenta un análisis sobre la economía del cambio climático, utilizando resultados de estudios científicos publicados y evaluados. Se encuentra estructurado en 6 partes que contienen 27 capítulos. La parte VI International Collective Action, que abarca desde el capítulo 21 al 27, trata, entre otros temas, de la coordinación de la acción colectiva internacional frente al cambio climático, que supone la existencia de un planificador central preocupado por el bienestar mundial y de la conveniencia de frenar la deforestación. Las consideraciones de este artículo se basan en la Acción Internacional Cooperativa, desarrollada en el Capítulo VI del Informe Stern, orientada a la implementación de las acciones necesarias para atenuar los efectos del cambio climático. Las acciones para afrontar los efectos adversos del cambio climático consisten en una amplia gama de actividades de distintos niveles y dimensiones para las cuales se requiere de un único marco de referencia internacional para reducir de manera efectiva la emisión de gases de efecto invernadero. Es así que los acuerdos internacionales para el cambio climático son la base fundamental para la cooperación; no obstante, existen retos inherentes en este aspecto debido a la naturaleza de la problemática, como son la provisión de un bien público como el clima y la atmósfera, el costo de la implementación de las acciones para el cambio climático, la transición de la economía global del carbono hacia una economía global limpia y la difusión pública de la importancia de participar en este proceso. La inversión, investigación e implementación de tecnologías para afrontar el cambio climático requieren de una planificación estratégica gubernamental adecuada que incluya reglas del juego firmes y que promuevan la adopción privada de acciones que reduzcan las emisiones de gases de efecto invernadero.

Palabras clave: cambio climático, calentamiento global, Informe Stern, efecto invernadero. 


\begin{abstract}
The Stern Review on the Economics of Climate Change, Stern Review on the Economics of Climate Change, is a document written by the economist Sir Nicholas Stern, a former World Bank member commissioned by the British Treasury, on the impact of climate change and warming global economy on the grounds that investment of $1 \%$ of world GDP is needed to mitigate the effects of climate change; otherwise the universe would be exposed to a recession that could reach $20 \%$ of PBI. It is a rigorous political report that managed to get public attention on the implications and consequences of climate change, leaving clear and conclusive conclusions about a prompt action. Their discussion Is about how to mitigate climate change and if we are prepared for this process from the economic. The report presents an analysis on the economy of Climate Change, using the results of scientific studies published and evaluated. It is structured in 6 parts that contain 27 chapters, part VI International Collective Action, which covers from chapter 21 to 27, deals, among other issues, with the coordination of international collective action against climate change, which presupposes the existence of a central planner concerned with global welfare and the desirability of curbing deforestation. The considerations of this article are based on the Cooperative Inter-Agency Action, developed in Chapter VI of the Stern Report, oriented to the implementation of the necessary actions to mitigate the effects of Climate Change. The actions to address the adverse effects of climate change consist of a wide range of activities of different levels and dimensions for which a single frame of reference is required to reduce the emission of greenhouse gases. Thus, international agreements for climate change are fundamental for cooperation, however, there are inherent challenges in this aspect due to the nature of the problem such as the provision of a global public good such as the climate and the atmosphere, the cost of implementation of actions for climate change, the transition of the global carbon economy towards a clean global economy and the public information on the importance of participating in this process. Investment, research and the implementation of technologies to address climate change require an adequate strategic planning that allows private actors to reduce their greenhouse gas emissions.
\end{abstract}

Key words: climate change, global warming, Stern review, greenhouse effect. 


\section{NOTA INTRODUCTORIA}

El cambio climático se ha vuelto un tema central en las relaciones internacionales de las últimas décadas, lo cual a su vez ha influenciado las políticas internas de los Estados y las relaciones sociales sobre problemas como la sostenibilidad del comercio, del desarrollo y el aseguramiento del recurso energético en el largo plazo. Una variedad de instituciones nacionales y acuerdos internacionales promueven las acciones paralelas y coordinadas sobre política energética, el uso de la tierra, el planeamiento estratégico, acciones de mitigación y la transición hacia una economía de bajo carbono. No obstante, existen retos, obstáculos y una problemática en su aplicación a una economía local y en desarrollo como el caso peruano. En ese sentido el presente artículo reflexivo describirá los retos de la implementación de acciones sobre el cambio climático de la manera que sigue:

I. Los desafíos de los acuerdos internacionales de cooperación para el cambio climático.

II. El Protocolo de Montreal como ejemplo de un acuerdo internacional exitoso.

III. Acciones para el cambio climático como provisión de un bien público global.

IV. Información pública sobre la necesidad de la implementación de las acciones para el cambio climático.

V. Transición hacia una industria energética limpia.

VI. Transición hacia una economía de bajo carbono

VII. La planificación estratégica y la creación de condiciones para la transición a una economía local limpia.

VIII. Incentivos e impuestos sobre acciones para el cambio climático.

IX. Inversión, investigación e implementación de tecnologías para afrontar el cambio climático.

En la primera parte, se describe brevemente el origen histórico de los acuerdos internacionales para el cambio climático, señalando cómo los acuerdos internacionales para la coo- 
peración sobre acciones para el cambio climático incluyen los siguientes desafíos que deben cumplir estos para lograr sus objetivos: (1) generar confianza, (2) ofrecer una adecuada distribución de los esfuerzos, y (3) deben ser llevados a cabo de manera consensuada con plena conciencia de la importancia del problema. En segundo lugar, se describe cómo el Protocolo de Montreal es definido como un ejemplo de un acuerdo internacional para la cooperación exitoso debido al consenso alcanzado y la información sobre la gravedad del problema del deterioro de la capa de ozono.

En la tercera parte, se analiza cómo la naturaleza de los efectos adversos del cambio climático sobre el clima, sobre la economía y sobre la sociedad es multidimensional y compleja. Es importante abordar brevemente la perspectiva que se usará al describir las acciones sobre el cambio climático y su problemática. El clima como elemento fundamental del medio ambiente es definido desde una perspectiva económica como un bien común, ${ }^{1}$ es decir, un bien sobre el cual nos beneficiamos todos los seres humanos independientemente de la nacionalidad, ubicación geográfica o estado socioeconómico; además, es indivisible y su uso o beneficio no puede ser restringido. El clima es el bien común más afectado debido al cambio climático, y sus efectos sobre la economía y el bienestar general son globales; por tanto, es un recurso importante que debe ser protegido. No obstante, surgen problemas de primera mano, como: ¿quién debería asumir los costos de las acciones de solución para detener los efectos adversos del cambio climático?, ¿cómo y en qué proporción se deberían distribuir estos costos entre todos los países?, ¿a nivel nacional y regional qué actor gubernamental o grupo social debería implementar o asumir la responsabilidad sobre estas acciones? Estas cuestiones se abordarán en la primera parte sobre los desafíos de los acuerdos internacionales.

La información al público general forma parte del consenso en la consecución de los objetivos y metas de las acciones para el cambio climático. Incrementar la comprensión del ciudadano de a pie es un requisito fundamental para generar confianza y compromiso con respecto a la implementación de políticas de gobierno sobre el cambio climático. La transparencia y la difusión de la evidencia científica son claves para justificar políticas e inversiones de largo plazo, como los impuestos a las emisiones o la promoción de las industrias limpias.

Otro eje fundamental se basa en la transición hacia una industria energética limpia o de bajo carbono debido a que es la base fundamental de una economía local o regional de bajas emisiones. La generación de energía es la actividad que más contribuye a las emisiones totales de gases de efecto invernadero, y como tal debe ser abordada de primera mano por los Estados. En ese sentido, se describe la problemática de la transición como las fallas de mercado que se producen por los subsidios a la generación de energía y el costo elevado de implementar una industria energética limpia frente a las fuentes tradicionales de uso intenso del carbono.

1 Luis Concha, Economía internacional (Lima: Universidad Inca Garcilaso de la Vega, 2015), 101. 
Consiguientemente, se explica la problemática de la transición hacia una economía de bajo carbono. Siendo que la medida fundamental y última para enfrentar el cambio climático es convertir la economía actual en una economía global de bajo carbono para reducir los efectos futuros negativos de la acumulación de gases de efecto invernadero, son los países en desarrollo los más vulnerables al cambio climático tanto por ser susceptibles a inundaciones, sequias o por tener recursos importantes en riesgo como la agricultura y la biodiversidad. ${ }^{2}$ Es así que para sentar las bases de esta transición se identifican tres retos aplicados al caso peruano, incluyendo (1) la creación de políticas de largo plazo, (2) la distribución y planificación de la propiedad de la tierra y (3) la inversión en gran escala para apoyar al sector privado en su transición hacia una economía local de bajo carbono.

La planificación estratégica es otro aspecto necesario y fundamental para la transición hacia una economía limpia. Esta, si bien incluye muchas de las acciones previamente descritas, incluye el enfoque de la planificación como un todo integrado a fin de dirigir una sola estrategia nacional. La planificación incluye la implementación de programas, la construcción de infraestructura, la inversión y desarrollo de tecnologías y el establecimiento de marcos regulatorios sólidos.

Los impuestos y los incentivos son mecanismos muy efectivos para lograr los objetivos de las acciones para el cambio climático. Se reconoce que los incentivos suelen ser en gran medida más eficientes al momento de promover actividades para el cambio climático como los subsidios, el financiamiento, la inversión pública y la rentabilidad de la implementación de tecnologías limpias y eficientes. Los impuestos por otro lado deben considerar la dinámica del mercado internacional, y es requisito que sean coordinados de manera conjunta para evitar la competencia desleal, la fuga de capitales y el traslado de las industrias.

Por último, se aborda el tema de la inversión, investigación e implementación de tecnologías para afrontar el cambio climático. Este tema está conformado por una serie de actividades en distintos niveles y una problemática compleja. Los retos más importantes giran en torno al financiamiento y el costo de adquirir e implementar las nuevas tecnologías de bajo carbono. ${ }^{3} \mathrm{El}$ marco legal juega un rol muy importante debido a que las inversiones para el cambio climático son de largo plazo y riesgosas en términos financieros por lo que es necesario que se establezcan reglas del juego claras, sólidas y que perduren a los cambios de Gobierno, y no solamente eso, sino que sean adoptadas multilateralmente para igualar las condiciones en el mercado internacional.

2 Moisés Aguilar, Practicas agroecológicas: para adaptarnos al cambio climático y mejorar nuestra producción (Lima: Instituto de Desarrollo y Medio Ambiente, 2016), 86.

3 Carlos Trinidad Alvarado, Opciones innovadoras del financiamiento de la conservación (Lima: Sociedad Peruana de Derecho Ambiental, 2017), 46. 


\section{LOS DESAFÍOS DE LOS ACUERDOS INTERNACIONALES DE COOPERA- CIÓN PARA EL CAMBIO CLIMÁTICO}

La respuesta internacional para el cambio climático proviene desde 1979, cuando se realizó la Primera Conferencia Mundial del Cambio Climático. En ella se resaltaron las preocupaciones de incremento del dióxido de carbono en la atmósfera. ${ }^{4}$ En 1988, la Asamblea General de las Naciones Unidas pone sobre la mesa una resolución, propuesta por Malta, a favor de la protección del clima para las presentes y futuras generaciones. En el mismo ańo, la Organización Meteorológica Mundial y el Programa Ambiental de las Naciones Unidas crearon el Panel Internacional para el Cambio Climático (IPCC). El IPCC publicó su Primer Reporte de Evaluación en 1990, confirmando que el cambio climático era una preocupación real y que las actividades humanas eran la causa más probable. ${ }^{5}$

El acuerdo internacional más importante actualmente es el Protocolo de Kioto, acordado en diciembre de 1997, el cual estableció una aproximación para el compromiso internacional de carácter vinculante y acordó compromisos específicos para el 2012. Este protocolo entró en vigor en febrero de 2005 y fue ratificado por 162 países. Sin embargo, EEUU y Australia se negaron a firmar el Protocolo y la administración canadiense negó poder cumplir con sus metas objetivos.

En ese marco, las acciones internacionales colectivas para proteger un bien público como la conservación del medio ambiente han ido incluyendo una amplia variedad de formas, incluyendo tratados específicos vinculantes como el Protocolo de Kioto y participación en sociedades y coaliciones regionales como las Naciones Unidas. Los acuerdos multilateral formales pertenecen al espectro de la cooperación y pueden brindar un alto grado de seguridad en el cumplimiento de las metas claves para implementar las acciones para el cambio climático a nivel internacional.

Entre las acciones para el cambio climático considerados en estos acuerdos se tienen acciones vitales como las reducciones en las emisiones de gases de efecto invernadero en todo el mundo. Sin embargo, existe una gama de acciones que incluyen acciones de mitigación y de prevención de mayores efectos negativos en el clima, como son la mejora de la salud, el empleo, la distribución de la renta, la mejora, la infraestructura industrial, el desarrollo regional directo, ${ }^{6}$ las inversiones estratégicas, las nuevas tecnologías, la reducción de emisiones,

4 Mónica Elizabeth Sánchez Vélez, Johannesburg Summit 2002: World Summit on Sustainable Development (Lima: United Nations, 2002), 3.

5 Robert Keohane, Instituciones internacionales y poder estatal: ensayos sobre teoría de las relaciones internacionales (Buenos Aires: Grupo Editor Latinoamericano, 1993), 78.

6 Ministerio del Ambiente del Perú, El Perú y el cambio climático: tercera comunicación nacional del Perú a la Convención Marco de las Naciones Unidas sobre el cambio climático (Lima: Ministerio del Ambiente, 2016), 120. 
la eficiencia energética, manejo y mejora de la conservación del ambiente, la producción de energía sostenible, el crecimiento económico local y nacional, la mejora del medio ambiente, la mejora de las tecnologías, reducción de la lluvia ácida y de otros contaminantes, los beneficios relacionados al cambio climático en el corto, mediano y largo plazo. Estos ejes son transversales y todos están dirigidos a abordar el tema de la mitigación de los efectos presentes del cambio climático, a la adaptación y a la prevención del incremento de los gases de efecto invernadero.

En ese sentido, los pactos internacionales sobre la cooperación internacionales para la implementación de las acciones para el cambio climático requieren de una serie de condiciones o desafíos para cumplir sus objetivos:

a) Confianza en los pactos internacionales: Los compromisos asumidos deben brindar confianza, y los mecanismos de cumplimiento deben ser proporcionados y adecuados. Debe primar la promoción y los incentivos frente a los castigos y penalidades.

b) Distribución de los beneficios y de los costos: Los acuerdos deben permitir acciones coordinadas incluso cuando un número de participantes perciba una desventaja, una imposibilidad de adoptar posiciones de liderazgo o una repartición desigual de compromisos, beneficios, responsabilidades o costos sobre las acciones de mitigación, adaptación o prevención. A diferencia de los tratados comerciales, los tratados para el cambio climático tienen beneficios difusos y de largo plazo, por lo que los beneficios político-económicos no son inmediatos.

c) Consenso internacional e información al público: ${ }^{7}$ Las acciones conjuntas deben ser motivadas por metas unilaterales que pueden fortalecerse por sí solas a través de un entendimiento común de la magnitud del desafío del cambio climático. La conciencia global sobre el cambio climático se debe hacer de manera multilateral, puesto que las sostenibilidades de estas políticas dependen de que los ciudadanos estén plenamente informados y estén dispuestos a participar y a asumir las responsabilidades civiles de las acciones para el cambio climático.

El primer desafío sobre la confianza y el respeto de los acuerdos internacionales es común a todos aspectos de las relaciones internacionales. Los Gobiernos se comprometen en acuerdos internacionales para la cooperación internacional del cambio climático porque se encuentran alineados con un sentido de responsabilidad y colaboración, y porque la opinión pública de sus respectivas naciones promueve tanto los objetivos trazados como los mecanismos para llegar a estos. Las nociones compartidas de un comportamiento responsable y colaborativo,

Finn Mogensen, Educación para el desarrollo sostenible: tendencias, divergencias y criterios de calidad (Barcelona: Grao, 2009), 32. 
dentro y fuera de los Gobiernos nacionales, crean condiciones para el cumplimiento de los compromisos internacionales.

Ejemplos exitosos de los acuerdos internacionales los encontramos en el marco de referencia de la convención del cambio climático de las Naciones Unidas y el Protocolo de Kioto. Ambos representan los principios fundamentales de una adecuada respuesta multilateral como solución al cambio climático.

El segundo desafío se refiere al problema de la distribución del esfuerzo, los costos de las acciones, de los beneficios y de los compromisos. No hay una fórmula única que pueda capturar de manera satisfactoria todos los aspectos pertinentes de una distribución equitativa de los esfuerzos entre los países a través de diversos criterios; sin embargo, existen marcos referenciales para la acción colectiva internacional que reconocen una restricción global de la cantidad a largo plazo sobre las emisiones a fin de distribuir equitativamente la responsabilidad de cumplir con el límite general entre los Estados. Cada uno de las acciones para el cambio climático descritas anteriormente conlleva sus propios desafíos específicos. Una respuesta eficaz al cambio climático requiere la cooperación en cada área, con el apoyo de una comprensión común de los objetivos a largo plazo, y la transparencia sobre la contribución de cada país.

Cuando tratamos el tercer desafío del consenso sobre la acción colectiva ${ }^{8}$ para el cambio climático nos referimos a una comprensión verdadera y compartida de la escala del desafío de la mitigación y la adaptación. Los Gobiernos por su parte deben conducir a un amplio consenso nacional sobre objetivos a largo plazo para la estabilización de los gases de efecto invernadero en la atmósfera, así como las consideraciones a más largo plazo sobre las vías apropiadas para la reducción de las emisiones mundiales, tales como la profundidad de las reducciones de emisiones para el presente siglo.

Brindar información pública sobre estos objetivos puede ayudar a dar claridad y facilitar el desarrollo de políticas nacionales e internacionales que minimicen los costos y maximicen los beneficios de la mitigación y la adaptación. ${ }^{9}$ Los responsables políticos pueden entonces ajustar la política nacional para operar en el contexto de un compromiso compartido de la acción colectiva internacional. Sin esto, existe el riesgo de que se generen compromisos fragmentarios o de corto plazo y se traduzcan en políticas inconsistentes que eleven los costos de acción y no tengan un impacto significativo en la reducción de emisiones.

El cambio climático ha tomado importancia en el trabajo de las Naciones Unidas y otras agencias (incluyendo el Programa Ambiental de la Naciones Unidas y la Organización de las

José Pinto-Bazurco Barandiarán, Diccionario internacional del medio ambiente (Lima: Titanium Editores, 2014), 212.

Antonio Ruiz Heredia, Educación, medio ambiente y didáctica del entorno: teoría y prácticas (Madrid: Popular, 2008 ), 120. 
Naciones Unidas para la Alimentación y la Agricultura) y sociedades (incluyendo el PROFOR, el programa de colaboración sobre bosques del Banco Mundial) que abordan el problema del uso de la tierra y de la agricultura. No obstante, los organismos internacionales tienen limitaciones en su campo de acción, por lo que los actores más apropiados son los Gobiernos nacionales y la ciudadanía.

\section{EL PROTOCOLO DE MONTREAL COMO EJEMPLO DE UN ACUERDO INTERNACIONAL EXITOSO}

El Protocolo de Montreal sobre las sustancias que disminuyen la capa de ozono es frecuentemente citado como el ejemplo de tratado internacional más exitoso para la cooperación internacional. Solo 24 países firmaron el Protocolo originalmente, en 1987; sin embargo, hacia octubre del 2006, el Protocolo ya contaba con 74 ratificaciones, incluyendo a los países desarrollados más importantes. La emisión de estos gases está bajo control y hay buenas señales de que la capa de ozono se recuperará en los próximos 100 años.

Muchos factores contribuyeron al éxito del Protocolo. Primero, hubo un alto grado de consenso científico y evidencia de que había un problema que requería acciones políticas inmediatas. El Protocolo estableció objetivos y metas para disminuir el uso de sustancias que disminuyen la capa de ozono, basados en recomendaciones de paneles expertos incluyendo Gobiernos y representantes de la industria. Segundo, aunque el consumo de los países en desarrollo era bajo, este aumentaba rápidamente. Los países en desarrollo participaron a raíz de los estudios científicos y del apoyo financiero que se les brindó para la adopción del tratado. Sin embargo, los resultados iniciales no eran buenos y el tiempo se agotaba. Tercero, Montreal reconoció la importancia de estimular y desarrollar nuevas tecnologías, de tal manera que la industria pudiera usar sustancias alternativas y proveer a los países en desarrollo de alternativas tecnológicas. Finalmente, se establecieron grupos de países ideológicamente similares en la discusión y análisis de las acciones y de las consecuencias del tratado.

Los acuerdos internacionales para la mitigación del cambio climático a diferencia de los acuerdos internacionales para controlar el deterioro de la capa de ozono son más complejos e involucran más dimensiones de la política internacional y diferentes enfoques de solución.

\section{LAS ACCIONES PARA EL CAMBIO CLIMÁTICO COMO PROVISIÓN DE UN BIEN PÚBLICO GLOBAL}

Reducir los riesgos del cambio climático es el ejemplo más representativo de la protección de un bien público global. Como se explicó en la parte introductoria, también es, en muchos sentidos, el ejemplo más puro de un bien público debido a que las emisiones de gases de efecto invernadero de cualquier país tienen el mismo efecto negativo en la atmósfera, la cual 
es un bien del que todos nos beneficiamos. El cambio climático también comparte algunas características con los desafíos ambientales que requieren de administración internacional de los recursos comunes, tales como la explotación de los bancos de peces, la protección de la capa de ozono y la provisión de bienes públicos globales en otras áreas, incluyendo la cooperación para el desarrollo y la salud. Mientras que el impacto de cambio climático es de una escala mucho más grande que la de estos ejemplos, hay mucho que se puede aprender de la experiencia de afrontar estos problemas. En este sentido, conforma un desafío importante determinar de manera efectiva el papel de los países industrializados, los países en desarrollo, los países más vulnerables y aquellos que más contribuyen a la emisión de los gases de efecto invernadero dentro de la estrategia internacional para la mitigación, adaptación y prevención de los efectos del cambio climático.

La mitigación del cambio climático es otro aspecto del problema primario de la provisión de un bien público global, así como la prevención de los efectos del cambio climático. La mitigación comparte algunas características importantes con los desafíos ambientales que requieren de una intervención internacional sobre recursos comunes para procurar que el mayor número de naciones contribuya con las acciones de control del cambio climático. Es importante que contribuyan en los acuerdos aquellos países que más se beneficiarían con estas acciones o que se encuentran en mayor peligro debido a su ubicación geográfica, como las costas, debido a inundaciones, o a su estructura económica, como los países agrícolas o con gran biodiversidad como el Perú. ${ }^{10}$

Para entender la manera de actuar de los Estados es importante considerar la dimensión económica del problema a través de la teoría de juegos. Mediante esta se argumenta que los individuos racionales actúan con interés propio y que no actuarían para asegurar un interés común a menos que se los coercione o se los induzca a realizarlo con incentivos que no estarían disponibles para aquellos que no participarán. Las acciones colectivas por parte de naciones soberanas es un desafío particular. En el tema del cambio climático no hay autoridad supranacional que pueda proveer acciones coercitivas, así que la cooperación requiere que las naciones perciban los suficientes beneficios para que estén dispuestos a participar en tratados y acuerdos internacionales, además de compartir una visión común del comportamiento responsable. Ellos deben también reconocer que, sin su involucramiento, las acciones colectivas podrían ser un fracaso.

10 Manuel Alberto Vizcarra Andreu, Ecósfera: la ciencia ambiental y los desastres ecológicos (Lima: ARS Asesoría y Servicios, 2016), 312. 


\section{INFORMACIÓN PÚBLICA SOBRE LA NECESIDAD DE LA IMPLEMENTA- CIÓN DE LAS ACCIONES PARA EL CAMBIO CLIMÁTICO}

El entendimiento masivo del público sobre el cambio climático y la promoción de soluciones está creciendo rápidamente, fortaleciendo de esta forma la toma de conciencia sobre sostener las acciones internacionales de cooperación para el cambio climático a través del tiempo. En este sentido, el desafío consiste en que las organizaciones gubernamentales y no gubernamentales capitalicen estas expectativas en acciones concretas para resolver los problemas de fondo sobre la reducción de emisiones y la transición de la economía global hacia una economía baja en carbono. Es difícil justificar una inversión a largo plazo o los impuestos a las emisiones sin un adecuado consenso público. ${ }^{11}$

Aumentar la comprensión del público sobre estas acciones en sus diferentes aspectos y niveles generará confianza entre los países con respecto a los esfuerzos de los demás, y esto podría fortalecer el esfuerzo general. El aumento de la información y el monitoreo pueden ayudar a que cada vez más individuos, organizaciones y Gobiernos contribuyan a esta causa y a mejorar la rendición de cuentas para la provisión de bienes públicos. En el caso del cambio climático, ya está claro que hay una serie de dimensiones, así como una variedad de enfoques que se superponen a la cooperación. Se requiere transparencia y una comprensión compartida de la acción colectiva a través de todos estos aspectos, en particular sobre la reducción de emisiones, el subsidio económico de industrias contaminantes, las políticas de inversión en innovación, la normas y regulaciones, los compromisos de la cooperación internacional en el despliegue y la difusión de estas tecnologías, así como el apoyo internacional para la adaptación al cambio climático.

La preocupación pública mundial y la conciencia sobre el cambio climático crecen rápidamente. Ambos influyen y sostienen la cooperación internacional, las aspiraciones nacionales y el liderazgo del sector privado en el cambio climático. En gran parte, debido a la evidencia científica y económica sobre el cambio climático que continúa acumulándose, así como el potencial negativo del cambio climático y las tecnologías futuras para sustituir a las de carbono. Es importante que la nueva información se refleje en las normas internacionales para la protección del clima, y que los políticos tengan claro cómo ajustarán sus objetivos a la luz de esta información, a fin de implementar acciones concretas de fondo.

\section{TRANSICIÓN A UNA INDUSTRIA ENERGÉTICA LIMPIA}

La generación de energía es la principal fuente de emisiones de gases de efecto invernadero, por lo que es vital enfocar los esfuerzos hacia esta actividad. Sin embargo, debemos contar con una adecuada implementación de políticas que promuevan industrias más limpias que

11 Santiago Álvarez, Economía ecológica; reflexiones y perspectivas (Madrid: Eds. Ciencias Sociales, 2009), 157 
brinden la apertura necesaria a oportunidades de negocio y mercados de energía de bajo carbono para una adecuada transición.

La energía tiene un papel fundamental en el desarrollo: ayuda a promover el acceso a una mejor educación, una mejor salud, una mayor productividad, una mayor competitividad y un crecimiento económico mejorado. En muchos países en desarrollo, la falta de inversión en infraestructura energética es un freno para el desarrollo, por lo que se suele realizar prácticas económicas como el subsidio a la generación eléctrica a través de la compra de los excedentes de la demanda o el subsidio directo.

En este contexto, debemos tener en cuenta la realidad de los países en desarrollo como el Perú al momento de considerar descarbonizar la generación de energía. En el Perú, la energía generada tanto de fuentes renovables como no renovables se efectúa en un contexto regulatorio especial. ${ }^{12}$ Lejos de incentivar el uso de energías limpias, en el Perú se subsidia el consumo de combustibles y diésel, y también se subsidia la generación de energía en un intento de mantener los precios finales al consumidor dentro de un margen aceptado por el público. Estas prácticas se deben eliminar puesto que distorsionan el mercado y lejos de incentivar la competencia y la transición a una economía limpia la favorecen y solidifican.

Es así que el Estado peruano y los usuarios de energía asumen el $100 \%$ de los costos de la generación de energía debido a que estos se dividen y actualizan en las tarifas; en ese sentido, la industria energética nunca tiene sobredemanda y no incurre en pérdidas. La razón sería que así se garantiza el suministro de energía a la industria y a la población en general; sin embargo, esto podría generar que la industria energética siga utilizando los mismos métodos contaminantes para la generación de energía como el carbón, el petróleo y el gas.

Por ello, el desafío consiste en transformar el mercado de la energía de manera progresiva superando estas fallas del mercado y coordinando con los sectores industriales presentes para eliminar el subsidio y sincerar el costo de la energía al consumidor final. Por ello, es importante contar con un marco regulatorio que no solo no favorezca a la energía intensa en la emisión de gases de invernadero, sino que la desincentive de manera racional y progresiva. Este primer paso allanará el camino para la promoción e incentivo de las energías limpias y podría ayudar a superar las fallas del mercado de capitales que conducen a la falta de inversión en eficiencia energética, y trabajar con el sector privado para aumentar la escala de la inversión con bajas emisiones de carbono. ${ }^{13}$

12 Arturo Vásquez Cordano, La industria de la energía renovable en el Perú: 10 años de contribuciones a la mitigación del cambio climático (Lima: Osinergmin, 2017), 115.

13 Gabriel Mejía Duclós, Experiencias de agricultura sostenible para afrontar los efectos del cambio climático (Lima: Instituto de Desarrollo y Medio Ambiente, 2017), 26. 
Otro reto importante es el costo de la energía renovable frente a las fuentes tradicionales de carbono. Si bien no sería posible reemplazar las energías intensivas en el uso de carbono al $100 \%$, se podrían realizar actividades de mitigación que incluyen la captura y el almacenamiento del carbono y mejorar la eficiencia energética de los procesos productivos reduciendo la cantidad necesarias de estas energías manteniendo el mismo nivel de producción. Es también necesario mencionar que no se debe aplicar impuestos a la generación de energía tradicional de carbono, puesto que lo único que se lograría es que las plantas de energía se trasladen a otros países donde no se apliquen estos impuestos. Es así que la promoción de las energías renovables debe primar por sobre el desincentivo.

\section{TRANSICIÓN HACIA UNA ECONOMÍA DE BAJO CARBONO}

Una de las soluciones de largo plazo para la conservación de los niveles de gases de efecto invernadero gira en torno a la transición de la economía global hacia una economía limpia. Se han determinado contradicciones como que los países más afectados por el cambio climático serían los países que cuentan con menos medios para afrontar sus efectos y aquellos con prioridades más urgentes dentro de sus presupuestos nacionales como el Perú. ${ }^{14}$ Las acciones para la adaptación al cambio climático incluyen la construcción de infraestructuras para la prevención de inundaciones como represas y la adopción de tecnologías para la agricultura en atención de las fluctuaciones climáticas como sequías y la escasez del agua. Siendo que estos proyectos son costosos en términos de un presupuesto nacional limitado, se esperaría que sus Gobiernos priorizaran políticas de salud, educación e infraestructura urbana por sobre acciones de mitigación o adaptación al cambio climático.

Estas presiones sobre la economía de los países en desarrollo se ven agravadas por la dificultad que enfrentan los Gobiernos nacionales y las autoridades locales para hacer cumplir las pocas regulaciones ambientales existentes o insistir en las inversiones en tecnologías nuevas de largo plazo. Estos factores pueden retrasar la introducción de tecnologías más eficientes que son comprobadamente rentables en los países desarrollado. Además, el nivel bajo de capacidad de oferta en relación con la demanda significa que es difícil para la industria que ponga sus plantas fuera de operación para hacer mejoras en la eficiencia energética y la distribución de energía, implicaciones que repercutirían directamente en la economía de los residentes locales y la propia industria. Por lo tanto, la infraestructura vieja de altas emisiones de carbono tiende a mantenerse en funcionamiento incluso cuando sería rentable para actualizarla.

14 Gerencia Regional de Recursos Naturales y Gestión del Medio Ambiente del Gobierno Regional de Lima, Estrategia regional de cambio climático de Lima (Lima: Gobierno Regional Lima, 2016), 20. 
En este sentido, el principal reto para la transición a una economía local y global limpia sería el liderazgo político económico para asumir los costos de la implementación de nuevas tecnologías de manera pública y guiar una transición adecuada y sostenible.

La cooperación internacional puede acelerar la innovación en tecnología de bajas emisiones y en tecnologías para la adaptación al cambio climático, puesto que existen muchos desafíos para reducir la emisión de los gases de efecto invernadero y resolver algunos de los principales retos para el desarrollo de políticas para afrontar el cambio climático:

- La creación de políticas de Estado de largo plazo para afrontar el cambio climático no son atractivas en términos de votos y campañas políticas, por lo que no forman parte central de la agenda política de nuestro país. ${ }^{15}$ Es necesario brindar más información al público en general sobre la necesidad de una inversión urgente en energías limpias, hábitos de consumo ecológicos y sobre la necesidad de políticas urgentes como impuestos a las emisiones y creación de estándares adecuados sobre la calidad de las emisiones. Esto generaría una carga fiscal y el aumento de precios de muchos productos, que, no obstante, deben ser asumidos hoy para evitar pérdidas mayores por los efectos del cambio climático.

- Actualmente, la distribución de la propiedad de la tierra en el Perú favorece más a la inversión en infraestructuras de corto plazo. Puesto que muchas inversiones están supeditadas a la negociación por el uso de la tierra y los conflictos sociales, los inversionistas deben tomar en cuenta la seguridad de sus proyectos a lo largo del tiempo. Debido a que las industrias y energía limpias son proyectos que requieren mucha inversión y tienen un tiempo de retorno largo, el Estado debe brindar la seguridad jurídica y fiscal de estas inversiones en favor de las más contaminantes: infraestructuras de mitigación del cambio climático, plantas de energía renovables, industrias de carbono "cero", etc. La planificación del uso de la tierra es vital para evitar conflictos en el desarrollo económico y urbano. ${ }^{16}$

- Se requieren inversiones a gran escala para apoyar la transición hacia una economía baja en carbono en los países en desarrollo como el Perú. Por ello, las instituciones financieras internacionales (IFI) tienen un papel importante que desempeñar para acelerar este proceso. Los Gobiernos nacionales deben coordinar con estos para recibir asistencia técnica para establecer políticas y marcos institucionales y así crear los incentivos adecuados en los sectores industriales pertinentes.

15 Sergio Federovsky, Historia del medio ambiente: la transformación de la naturaleza, del mundo ajeno y amenazante al espacio a conquistar; La génesis del movimiento ambientalista (Buenos Aires: Capital Intelectual, 2007), 61.

16 Instituto Nacional de Estadística e Informática, Perú: Anuario de estadísticas ambientales, 2016 (Lima: INEI, 2017$), 112$. 
Está claro que el cambio climático es ahora un problema importante para el crecimiento económico y el desarrollo, y debe considerarse dentro de las estrategias de asistencia de los países desarrollados. La demanda de energía y el uso de vehículos diésel está creciendo rápidamente en el Perú. La inversión que se realice en los próximos 10-20 ańos podría bloquear en emisiones muy altas para el próximo medio siglo, o presentar una oportunidad para mover la economía hacia un camino más sostenible. ${ }^{17}$

La inversión en la eficiencia energética puede reducir el crecimiento de la demanda, y las tecnologías de baja emisión de carbono pueden reducir aún más el impacto sobre el cambio climático. Un eje importante constituye el cómo la transición a una economía global de bajo carbono puede ser acelerada a través de acciones para promover la difusión de tecnologías e inversión en infraestructura de bajo carbono en países en desarrollo y economías en transición.

Es posible que se requieran diferentes intervenciones de política en cada etapa de esta transición, según las funciones que los mercados privados puedan proporcionar con éxito. Las intervenciones de política varían según la naturaleza de la tecnología, su etapa de desarrollo comercial y las características políticas y económicas de los países proveedores y receptores, las cuales un incluyen un proceso de implementación por pasos.

a. Evaluación de las necesidades tecnológicas.

b. Selección de tecnologías.

c. Mecanismos para la importación de tecnología.

d. Escala de la implementación de la tecnología.

e. Adaptar la tecnología a las condiciones locales.

f. Mejorar la infraestructura instalada.

g. Desarrollo y mejoramiento de la tecnología existente.

Para ser sostenible, la transferencia de tecnología debe ser parte de un proceso más amplio de transferencia de la capacidad de creación de tecnológica a los países en desarrollo. El desarrollo de la capacidad tecnológica depende de la transferencia de habilidades, conocimientos y experiencia, así como de hardware, especialmente si las tecnologías deben ser asimiladas y desarrolladas en los países receptores de estas tecnologías. El desarrollo de capacidades y conocimientos debe adaptarse a las circunstancias locales, porque la falta de habilidades técnicas, comerciales o regulatorias resultaría en un intento fallido de transferencia de tecnología. La transferencia de tecnología también se debe enfocar en la creación de una competencia mejo-

17 Gladys Terraza, Medio ambiente y acceso a la propiedad y al territorio (Lima: La Paz, 2016), 99. 
rada y accesible en servicios asociados, conocimiento organizacional y un marco regulatorio apropiado para fortalecer y coordinar las redes a través de las cuales las partes interesadas facilitan la transferencia.

\section{LA PLANIFICACIÓN ESTRATÉGICA Y LA CREACIÓN DE CONDICIO- NES PARA LA TRANSICIÓN A UNA ECONOMÍA LOCAL LIMPIA}

Es probable que las reducciones de emisiones a gran escala más rentables estén relacionadas con programas estratégicos, por ejemplo, apoyando en la implementación de sistemas integrados de transporte, transporte masivo para usuarios y desarrollo urbano, o abordando una transición mayorista hacia una generación de energía con menos carbono, incluida la adaptación de plantas ineficientes y el uso sistemático de captura y almacenamiento de carbono. Los programas en esta escala solo pueden tener lugar en el contexto de reformas estructurales y políticas de desarrollo implementadas por Gobiernos nacionales o regionales. La inversión en proyectos para la mitigación, adaptación o prevención del cambio climático tiende a dirigirse a países donde existe un entorno favorable para la inversión del sector privado (por ejemplo, estabilidad económica y política, mercados libres de intervención, estructuras legales sólidas) y países que han desarrollado la capacidad nacional para utilizar esta fuente de financiación. Esto proporciona sólidos incentivos para que los países desarrollen dichos entornos. ${ }^{18}$

La planificación estratégica a largo plazo también es esencial para ofrecer la infraestructura de desarrollos sostenibles en el sector del transporte. En ese sentido, la política pública también tiene una gran influencia en la creación de las condiciones para el sector privado a invertir, e incentivar y transferir tecnologías de bajo carbono (y las tecnologías pertinentes para la adaptación) a los países en desarrollo. Es importante entender las diversas funciones de la protección de los derechos de propiedad intelectual, puesto que un marco legal poco sólido en términos de patentes y derechos de propiedad intelectual podría desincentivar la investigación y desarrollo de nuevas tecnologías limpias. ${ }^{19}$

\section{INCENTIVOS E IMPUESTOS SOBRE ACCIONES PARA EL CAMBIO CLIMÁTICO}

Es importante reconocer que los países tienen diferentes preferencias, instituciones y tradiciones. Estas afectan a las elecciones que hacen los Gobiernos entre los instrumentos de política, tales como impuestos, el comercio, la regulación y los subsidios, además de los enfo-

18 Deniss Malpica Alfaro, Técnicas y estrategias andinas de adaptación al cambio climático: manual de capacitación e implementación (Cajamarca: Appleton Foundation, 2017).

19 Arístides Sotomayor, Tecnologías limpias: medio ambiente y comercialización de minerales (Lima: Universidad de Lima, 2017), 523. 
ques voluntarios y obligatorios. Un desafío clave para los marcos internacionales es permitir la adecuada acción multilateral y paralela en diferentes países, a fin de gestionar y coordinar las interacciones entre los diferentes enfoques nacionales. Se debe reconocer que los mecanismos de obediencia que confían en duros castigos son difíciles de hacer cumplir, porque frecuentemente tienen un detrimento tanto en el que impone los castigos como en el sujeto que incumple, y crean incentivos en ambos para ignorar los acuerdos.

Las reducciones de la emisión de gases de efectos invernadero en esta escala podrían ser logradas facilitando políticas que reduzcan el costo de las acciones para afrontar el calentamiento global y que promuevan una distribución equitativa de la responsabilidad. Los mercados globales de uso intensivo de carbono pueden ser dirigidos a crear los precios y canales de inversión hacia una economía de bajo carbono tanto en países ricos como los países pobres. Los países están motivados a participar en la cooperación internacional para el cambio climático por una serie de razones, incluyendo la promoción de una variedad de objetivos de corto plazo, así como de largo plazo, para reducir los riesgos del cambio climático.

Existen compañías internacionales líderes que muestran cómo se pueden generar ganancias al reducir las emisiones de las actividades industriales globales. Cada vez más compañías multinacionales son responsables de sus operaciones alrededor del mundo, y un número cada vez más grande de líderes de negocios preferirían ver un marco político de largo plazo para planificar de manera adecuada y global. Compañías de gran escala poseen horizontes de tiempo más amplios que las escalas de tiempo gubernamentales y realizan sus propias proyecciones sobre los efectos de las futuras políticas, basadas en parte en sus perspectivas de la presente y futura opinión pública. En este sentido, un primer reto consiste en crear un marco sólido internacional y nacional que permita que las compańías internacionales puedan realizar inversiones sobre acciones para el cambio climático sin riesgos en sus inversiones debido a cambios de Gobierno o modificaciones en las reglas de juego que los perjudiquen. Debemos buscar promover e incentivar la participación del sector privado para contribuir a reducir la emisión de los gases de efecto invernadero e invertir en el uso de tecnologías limpias.

Un incentivo natural de mercado consiste en que los negocios son motivados cada vez más por oportunidades de reducir costos a través del aumento de la eficiencia energética y a través de una proyección inteligente de los mercados futuros, como por ejemplo el desarrollo de automóviles híbridos por algunos fabricantes de automóviles. Un gran número de compañías multinacionales en varios sectores industriales, incluyendo el automotor, el energético, los intensivos en energía; y los financieros han empezado a identificar estrategias para una sociedad de "cero" carbón sin necesidad de incentivos adicionales por parte de los Gobiernos. Esto abre las puertas al desafío del incentivo y promoción de la proliferación de este tipo de negocios a 
través de reducción de impuestos a las industrias limpias, el apoyo al financiamiento de estos negocios y otros de carácter gubernamental. ${ }^{20}$

Por otro lado, los impuestos, a diferencia de los incentivos, tienen menos ventajas a nivel internacional y requieren de un enfoque internacional coordinado basado en impuestos para que los países puedan tomar sus decisiones fiscales de forma individual y evitar la fuga de inversiones hacia países con distintas regulaciones tributarias. No obstante, esto requiere de estructuras e instituciones elaboradas cuya constitución puede llevar tiempo y esfuerzo.

En este sentido, un desafío importante está conformado por la armonización internacional de los impuestos sobre el carbono, lo cual puede ser extremadamente difícil en la práctica. En países europeos, los acuerdos sobre un impuesto al carbono común han fracasado previamente, terminando con un complejo mosaico de aplicación parcial y excepciones tributarias dentro de los países. Es más, buscar un impuesto uniforme a nivel internacional impediría la libertad nacional sobre la forma de aplicar los objetivos ambientales; y esto puede entrar en conflicto con la soberanía nacional y la política práctica de la formación de la política interna.

Otra perspectiva de los impuestos incluye la aplicación de los impuestos no sobre las industrias sino sobre las emisiones domésticas, o en el contenido de carbono en el comienzo de la cadena de suministro de los bienes de consumo, evitando así la reubicación y fuga de inversiones y la competencia desigual en la industria de la generación de energía. En realidad, este tipo de impuestos son mitigados sustancialmente por los costos de reubicación y muchos otros factores que influyen en el grado de competitividad que las empresas enfrentan, la elasticidad y precio de la demanda de productos, así como la estructura de mercado. ${ }^{21}$

\section{INVERSIÓN, INVESTIGACIÓN E IMPLEMENTACIÓN DE TECNOLO- GÍAS PARA AFRONTAR EL CAMBIO CLIMÁTICO}

La inversión, investigación e implementación de nuevas tecnologías para afrontar el cambio climático comprenden la inversión en plantas industriales, estaciones productoras de energía, infraestructura vial, etc., que sean bajas en el uso de carbono y por tanto que reduzcan la cantidad de emisiones de gases de carbón. A diferencia de la mitigación, la inversión en tecnologías limpias tendría efectos de largo plazo; es así que todo lo que se implemente en estas décadas podría tener efectos positivos en la segunda mitad de este siglo. Pero no solamente eso, sino que actualmente las tecnologías bajas en carbono son más costosas que las

20 María Novo Villaverde, La interpretación de la problemática ambiental: enfoques básicos (Madrid: Fundación Universidad Empresa, 1997), 88.

21 Rocío Mónica Vera Herrera, Ecología \& medio ambiente: compendio teórico-práctico (Lima: Servicios Gráficos Gonzales, 2017), 115. 
tradicionales, haciendo estas inversiones inciertas y poco atractivas si no se cuenta con una política internacional clara y de largo plazo.

Entre los medios para financiar la implementación de nuevas tecnologías de bajo carbono se incluyen el promover una mayor inversión pública y privada en el desarrollo de energía de bajo carbono, y en establecer vías de cooperación para crear estándares de bienes y servicios alineados en la transición a una economía de bajo carbono.

En este sentido, para financiar la inversión en tecnologías de bajo carbono se deben establecer políticas nacionales claras. Una de las estrategias más directas es a través del impuesto a las emisiones de carbono. Los impuestos elevarían el costo de las actividades intensivas en el uso del carbono, haciendo las tecnologías limpias más atractivas para el sector privado, y con el monto fiscal recaudado se podría seguir promoviendo el desarrollo de tecnologías de procesos productivos menos contaminantes y energías más limpias.

Esto requiere de políticas sólidas que se mantengan el tiempo que justifiquen la financiación de tecnologías de bajo carbono y la transformación de la economía a una más limpia. Estas políticas deben incluir una información precisa sobre los cambios en el clima y anticipación de riesgos como desastres naturales, así como una planificación del uso de la tierra para fines industriales y urbanos con estándares medioambientales favorables, la protección de los recursos naturales susceptibles por los efectos del cambio climático y el aseguramiento de los sectores de la población más vulnerables como el sector agrario. ${ }^{22}$

Sin embargo, estas políticas no se pueden tomar unilateralmente, sino que se deben adoptar en todos los países. Si estos impuestos se imponen individualmente en un determinado país, las industrias y las fuentes de energía se podrían trasladar hacia otros países que no promueven la reducción de las emisiones de gases de carbono. La adecuada implementación de estas políticas de manera colectiva debería abrir el camino hacia una economía global de bajo carbono.

\section{REFLEXIONES FINALES}

El desafío clave es formar un acuerdo o un grupo de acuerdos que atraigan la participación de todos los países con el objetivo de reducir significativamente la emisión de gases de invernadero y lograr una reducción sostenible en todos los sectores. A medida que la ciencia del cambio climático sea ampliamente aceptada, las actitudes públicas harán cada vez más difícil para los líderes políticos de todo el mundo minimizar la importancia de una acción decisiva para responder al desafío.

22 Berenice Cajavilca Gonzales, Modelos económicos de desarrollo productivo y tecnológico (Lima: Universidad Inca Garcilaso de la Vega, 2016), 72. 
Al final, lo que importa es que el esfuerzo global coincida con la magnitud del problema, que las partes perciban confianza en que la distribución del esfuerzo sea justa, que vaya de la mano con la eficiencia y que la opinión pública sea favorable y sea capaz de mantener la cooperación en esos términos en el largo plazo. Es urgente crear un marco regulatorio internacional que brinde una respuesta eficiente y equitativa para la acción colectiva internacional.

Las formas en que la cooperación es evaluada deber ser amplia, de la misma manera que los métodos utilizados para la gestión del rendimiento de las empresas se han ampliado en los últimos años a través del uso de métodos matemáticos, estadísticos y financieros. La tarea de la evaluación comparada de la acción responsable de otros países se hace más complicada en el caso del cambio climático debido a los horizontes de tiempo y los efectos de la implementación de las acciones de mitigación. La prevención, no obstante, es necesaria.

Mayores acciones son necesarias para estabilizar las concentraciones de gases de invernadero en la atmósfera. Acciones exitosas en muchas áreas, incluyendo la protección de la capa de ozono y el Protocolo de Montreal, han demostrado que la cooperación internacional puede sobrellevar problemas como la distribución desigual de la responsabilidad y de los costos asociados. Aportes de la teoría de juegos brindan mayor información sobre el diseño de marcos de referencia para la acción internacional.

La adopción de medidas para reformar el sector de la energía puede ser difícil, pero a medida que se eliminan las distorsiones subyacentes en los precios de la energía y los subsidios, surgirán las tecnologías eficientes y de baja emisión de carbono económicamente rentables. Esto también puede aumentar la inversión pública, así como la inversión extranjera. El mejoramiento de la eficiencia energética también puede aprovechar las oportunidades de ganancias significativas mediante la eliminación de las tecnologías de generación obsoletas, reducción de las pérdidas en la transmisión de energía, y la mejora de los efectos positivos de la eliminación intensiva de carbono y de los combustibles contaminantes.

En ese sentido, el siguiente paso es la transición a una economía global de bajo carbono que conlleva desafíos importantes como el costo de la implementación de tecnologías limpias, el poco interés político o la falta de liderazgo para impulsar proyectos de largo plazo que transformen la economía. La planificación estratégica juega un rol muy importante al integrar todos los requisitos hacia una transición sostenible y eficiente, estableciendo políticas nacionales claras y sólidas sobre las cuales puedan realizar inversiones seguras de largo plazo y promover el financiamiento privado o público de las acciones para afrontar los efectos negativos del cambio climático. Por otra parte, debemos buscar promover e incentivar la participación del sector privado para contribuir a reducir la emisión de los gases de efecto invernadero e invertir en el uso de tecnologías limpias. 


\section{REFERENCIAS}

- Aguilar Huamaní, Moisés. Practicas agroecológicas: para adaptarnos al cambio climático y mejorar nuestra producción. Lima: Instituto de Desarrollo y Medio Ambiente, 2016.

- Álvarez, Santiago. Economía ecológica; reflexiones y perspectivas. Madrid: Eds. Ciencias Sociales, 2009.

- Cajavilca Gonzales, Berenice. Modelos económicos de desarrollo productivo y tecnológico. Lima: Universidad Inca Garcilaso de la Vega, 2016.

- Concha Sequeiros, Luis. Economía Internacional. Lima: Universidad Inca Garcilaso de la Vega, 2015.

- Federovsky, Sergio. Historia del medio ambiente: la transformación de la naturaleza, del mundo ajeno y amenazante al espacio a conquistar; La génesis del movimiento ambientalista. Buenos Aires: Capital Intelectual, 2007.

- Gerencia Regional de Recursos Naturales y Gestión del Medio Ambiente del Gobierno Regional de Lima. Estrategia regional de cambio climático de Lima. Lima: Gobierno Regional Lima, 2016.

- Instituto Nacional de Estadística e Informática. Perú: Anuario de estadísticas ambientales, 2016. Lima: INEI, 2017.

- Keohane, Robert. Instituciones internacionales y poder estatal: ensayos sobre teoria de las relaciones internacionales. Buenos Aires: Grupo Editor Latinoamericano, 1993.

- Malpica Alfaro, Deniss. Técnicas y estrategias andinas de adaptación al cambio climático: manual de capacitación e implementación. Cajamarca: Appleton Foundation, 2017.

- Mejía Duclós, Gabriel. Experiencias de agricultura sostenible para afrontar los efectos del cambio climático. Lima: Instituto de Desarrollo y Medio Ambiente, 2017.

- Ministerio del Ambiente del Perú. El Perú y el cambio climático: tercera comunicación nacional del Perú a la Convención Marco de las Naciones Unidas sobre el cambio climático. Lima: Ministerio del Ambiente, 2016.

- Mogensen, Finn. Educación para el desarrollo sostenible: tendencias, divergencias y criterios de calidad. Barcelona: Grao, 2009.

- Novo Villaverde, María. La interpretación de la problemática ambiental: enfoques básicos. Madrid: Fundación Universidad Empresa, 1997. 
- Pinto-Bazurco Barandiarán, José. Diccionario internacional del medio ambiente. Lima: Titanium Editores, 2014.

- Ruiz Heredia, Antonio. Educación, medio ambiente y didáctica del entorno: teoría y prácticas. Madrid: Popular, 2008.

- Sánchez Vélez, Mónica Elizabeth. Johannesburg Summit 2002: World Summit on Sustainable Development. Lima: United Nations, 2002.

- Sotomayor, Arístides. Tecnologías limpias: medio ambiente y comercialización de minerales. Lima: Universidad de Lima, 2017.

- Terraza, Gladys. Medio ambiente y acceso a la propiedad y al territorio. Lima: La Paz, 2016.

- Trinidad Alvarado, Carlos. Opciones innovadoras del financiamiento de la conservación. Lima: Sociedad Peruana de Derecho Ambiental, 2017.

- Vásquez Cordano, Arturo. La industria de la energía renovable en el Perú: 10 años de contribuciones a la mitigación del cambio climático. Lima: Osinergmin, 2017.

- Vera Herrera, Rocío Mónica. Ecología \& medio ambiente: compendio teórico-práctico. Lima: Servicios Gráficos Gonzales, 2017.

- Vizcarra Andreu, Manuel Alberto. Ecósfera: la ciencia ambiental y los desastres ecológicos. Lima: ARS Asesoría y Servicios, 2016.

\section{FUENTES HEMEROGRÁFICAS}

http://www.eleconomistaamerica.pe/economia/noticias/245687/07/07/Informe-Stern-la-verdad-del-cambio-climatico.html

https://es.wikipedia.org/wiki/Informe_Stern

http://www.ambientum.com/documentos/general/resumeninformestern.pdf

Recibido: $12 / 01 / 2018$

Aprobado: 13/04/2018 